\title{
MONITORING SUB-WEEKLY EVOLUTION OF SURFACE VELOCITY AND ELEVATION FOR A HIGH-LATITUDE SURGING GLACIER USING SENTINEL-2
}

\author{
Bas Altena $^{1 *}$, Odin Næss Haga ${ }^{1}$, Christopher Nuth $^{1}$, Andreas Kääb $^{1}$ \\ ${ }^{1}$ Department of Geosciences, University of Oslo, 0371 Oslo, Norway \\ (bas.altena, odinh, christopher.nuth, kaeaeb)@ geo.uio.no
}

Commission III, WG III/9

KEY WORDS: Glaciology, pushbroom photogrammetry, time-series, surface kinematics, Negribreen, Svalbard

\begin{abstract}
:
Currently, the Sentinel-2 twin satellite constellation of the Copernicus program is in operational mode and generates high repeat acquisitions at high-latitudes during polar day. These pushbroom satellites have a large field-of-view and are therefore ideal for simultaneous extraction of glacier displacement and elevation data. In this study we showcase the capabilities of this system set-up by generating time-series of glacier flow and elevation change over Negribreen, a tidewater glacier in Svalbard which nowadays is in its surge phase.
\end{abstract}

\section{INTRODUCTION}

The Sentinel-2 twin satellite constellation is part of the operational Copernicus monitoring system, and acquires medium resolution imagery with a wide field-of-view of 270 kilometers over the Earth surface for most of its sun-lit orbit. For only one satellite, its acquisition coverage at the equator resolves in a repetition rate of 10 days. Convergence of the orbits towards the poles cause this repetition rate to increase, as can be seen in Figure 1. Consequently, locations at high latitudes are observed from different angles, making across-track photogrammetry possible. This potential of Sentinel-2 has been demonstrated before by non-orthorectified images (Gaudel et al., 2017, Lacroix et al., 2018), but was limited to the calculation of a single elevation model.

The scope of this study is to introduce an approach that can exploit such highly redundant datasets at high latitudes, for example to monitor rapid glacier dynamical change. We use a surging glacier in Svalbard to demonstrate this potential because a glacier surge has both strong lateral and vertical changes within a short time period.

A glacier surge involves a rapid increase of glacier velocity, which can be several orders of magnitude faster than during its quiescent phase (Cogley et al., 2011). Surge events are accompanied by large transfer of ice mass from the accumulation area of the glacier towards lower elevations, often resulting in glacier terminus advance. In Svalbard a large fraction of the glaciers are of surge-type (Hamilton , Dowdeswell, 1996). Such classifications come from the observation that surge events seem to occur episodically on multiple glaciers. Sometimes the interval is semi-periodic (Eisen et al., 2005), but because of the long separation time (which can be in the order of several decades), not much is known about surges characteristics or changes thereof. When sufficient topographic data is available, the build-up of mass in the accumulation area during quiescence can be detected through elevation differencing and indicate surge susceptibility

\footnotetext{
${ }^{*}$ Corresponding author: Bas Altena, bas.altena@geo.uio.no
}

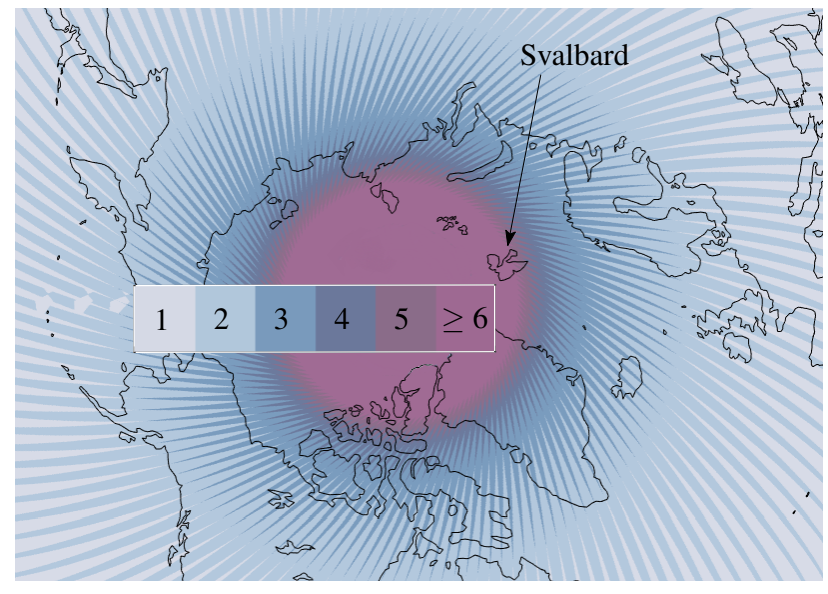

Figure 1. Illustration of the amount of times a point can be seen from the different orbits of Sentinel-2.

beforehand (Sund et al., 2009).

Recently a large glacier on Olav V Land in Northeast Spitsbergen has started to surge (Strozzi et al., 2017). The Negribreen glacier system has surged at least twice before, around 1870 and in 1935 (Lefauconnier, Hagen, 1991). Knowledge about specific characteristics of these previous surges is limited, though recent multibeam bathymetry in front of the glacier reveals the surge extent of the last event seems to have overridden the previous surge remnants (Ottesen et al., 2017).

In this study we investigate the possibility of simultaneously extracting horizontal velocity and vertical elevation change through time from a large collection of repeat multi-angle satellite images. Both parameters are partially independent, as the surface displacement may not reflect the topographic bulge of mass transfer (sometimes described as kinematic wave) often associated with surge events. During a surge, both of these processes may occur at different velocities (Raymond, 
1987). Hence, this makes the formulation photogrammetrically time-dependent and multi-temporal, as the two signals are super-imposed. To our knowledge this is the first demonstration of such a set-up, but is within the same family of work as (Kaufmann , Ladstädter, 2004, Li et al., 2017), though with different base data and assumptions.

\section{DATA}

A total of 246 images from the Sentinel-2 archive over Negribreen where manually selected and these are (partly) cloud free and taken during the polar days of 2016, 2017 or 2018. Data is acquired from eight unique relative orbits due to orbit convergence close to the poles (see Table 1), which produces redundant images taken from different zenith distances and with varying satellite bearings (azimuth). Displacements are matched between all possible pairs of cloud-free images that are within 100 days time separation. The network of displacements can be separated into three distinct graphs, because of the polar night and the limit of maximum desired time separation. This results in a total of 7763 displacement fields (see Figure 2) and groups of 1070, 2866, 3827 for the different graphs of 2016 to 2018, respectively. The increase in pairs over time is related to the launch of Sentinel-2B on the $23^{\text {rd }}$ Jan. 2015, its transfer from commissioning phase towards operational and the start of the data transfer through the European Data Relay System.

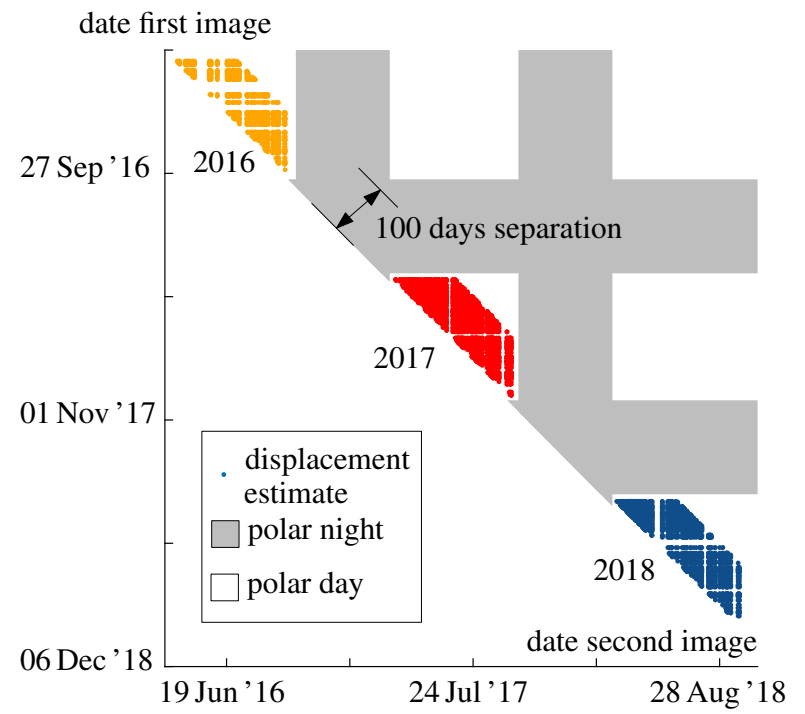

Figure 2. Temporal distribution of velocity fields. The shaded gray regions denotes the polar night when optical imagery is not available. Because of the limited timespan of 100 days, three distinct time periods are formed.

Sentinel-2 data is available in orthorectified form, and are orthorectified using (by necessity) an outdated elevation model (DEM) that does typically not represent glacier surfaces correctly due to their significant changes over time or steep topography(Kääb et al., 2016, Altena , Kääb, 2017, Ressl , Pfeifer, 2018). Therefore, Sentinel-2 data can be considered to be pseudo-orthophotos (Kaufmann, Ladstädter, 2004) for the application of glacier monitoring. Here we use standard image matching procedures (Leprince et al., 2007), in a pyramid structure and with an intermediate post-processing procedure
(Westerweel, Scarano, 2005). The search range applied is 2560 meters ( $2^{8}$ pixels), has three layers where the final template size is 320 meter ( $2^{5}$ pixels), which is also the separation distance between different templates.

\begin{tabular}{|ccc|}
\hline orbit & $\begin{array}{c}\text { bearing } \\
\phi[\mathrm{deg}]\end{array}$ & $\begin{array}{c}\text { zenith } \\
\theta[\mathrm{deg}]\end{array}$ \\
\hline 009 & -136.4 & -2.1 \\
038 & -126.9 & +7.5 \\
052 & -134.0 & +0.4 \\
066 & -141.1 & -7.8 \\
081 & -124.5 & +9.6 \\
095 & -131.7 & +2.9 \\
109 & -138.7 & -4.9 \\
138 & -129.3 & +5.3 \\
\hline
\end{tabular}

Table 1. Mean acquisition angles over Negribreen from different relative Sentinel-2 orbits.

\section{METHODOLOGY}

Here we adopt the formulation of pushbroom photogrammetry as in (Altena , Kääb, 2017) but extend parameter estimation with a time dependent elevation bias. In this way the vertical difference and horizontal displacement can be formulated as,

$$
\begin{aligned}
& \underbrace{\left[\begin{array}{cc|cc}
\delta t_{p q} & 0 & \tan \theta_{p} \cdot \cos \phi_{p} & -\tan \theta_{q} \cdot \cos \phi_{q} \\
0 & \delta t_{p q} & \tan \theta_{p} \cdot \sin \phi_{p} & -\tan \theta_{q} \cdot \cos \phi_{q}
\end{array}\right]}_{\left[\mathbf{A}_{p q} \mid \mathbf{C}_{p} \mathbf{C}_{q}\right]} \\
& \underbrace{\left[\begin{array}{c}
\vec{v}(x)_{p q} \\
\vec{v}(y)_{p q} \\
\delta h_{p} \\
\delta h_{q}
\end{array}\right]}_{\mathbf{x}}=\underbrace{\left[\begin{array}{c}
\vec{d}(x)_{p q} \\
\vec{d}(y)_{p q}
\end{array}\right]}_{\mathbf{y}} .
\end{aligned}
$$

$$
\begin{array}{ll}
\text { where } & \vec{d}=\text { displacement measurement } \\
\delta t=\text { time interval } \\
\vec{v}=\text { velocity estimate } \\
\phi=\text { satellite bearing } \\
\theta=\text { zenith distance } \\
\delta h=\text { vertical orthorectification bias } \\
x, y=\text { spatial coordinates } \\
t=\text { temporal coordinate } \\
p, q=\text { time instances }
\end{array}
$$

In this formulation the system of equations is ill-posed; there are more unknowns than measurments. When four images are included and all are matched with each other, then theoretically, this system can be solved $(n(n-1)>2 n-1$ where $n$ is the amount of images). Hence, the construction of the equations given in Eq. 1 can be placed into a larger matrix system as follows,

$$
\left[\begin{array}{c}
\vec{d}_{p q} \\
\vec{d}_{q r} \\
\vec{d}_{p r}
\end{array}\right]=\left[\begin{array}{ll|lll}
\mathbf{A}_{p q} & 0 & \mathbf{C}_{p} & \mathbf{C}_{q} & 0 \\
0 & \mathbf{A}_{q r} & 0 & \mathbf{C}_{q} & \mathbf{C}_{r} \\
\mathbf{A}_{p r} & \mathbf{A}_{p r} & \mathbf{C}_{p} & 0 & \mathbf{C}_{r}
\end{array}\right]\left[\begin{array}{c}
\vec{v}_{p q} \\
\vec{v}_{q r} \\
\delta h_{p} \\
\delta h_{q} \\
\delta h_{r}
\end{array}\right]
$$




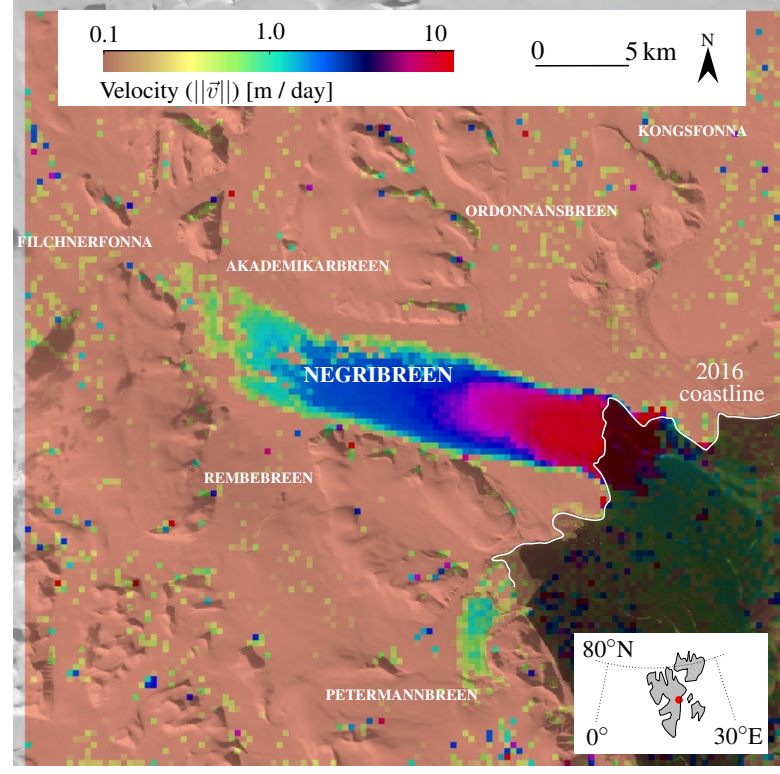

Figure 3. Estimated daily velocity of the Negribreen glacier system for April 2018.

Recently time-series of displacement estimates from optical remote data has moved from pair-wise snapshot analysis towards network inversion (Bontemps et al., 2018, Altena et al., 2019), with networks formulated as above (though neglecting the geometric component $\mathbf{C}$ ). However, the variance of the displacement estimates are non-Gaussian with heavy tails, consequently ordinary least squares estimation is not practical. When in addition the redundancy number is low, post-processing based on consensus voting might be best (Altena et al., 2019). While with a well connected network of displacements implementation of more advanced robust solvers is possible (Hadhri et al., 2019). Though for this show case, the measurement vector is substantially large $(n>1000)$, as well as, the amount of unknown parameters, which is unfavorable for least squares adjustment, as large matrices need to be inverted. Furthermore, a considerable amount of noise can be present, thus forcing one to use costly optimization methods. For these reasons we applied an adapted RANSAC (Fischler , Bolles, 1981) procedure instead. However, one downside of this methodology is that it hampers the possibility to weight the adjustment through, for example, their time separation.

\section{RESULTS}

Negribreen $\left(78.5^{\circ} \mathrm{N} 19.1^{\circ} \mathrm{E}\right)$ is a $964 \mathrm{~km}^{2}$ polythermal tidewater glacier in which a large portion of the glacier bed is located below sea level (Dowdeswell et al., 1984). The glacier tongue was stagnant prior to 2016 as horizontal velocity is practically inexistent. This suggests the tongue may have been frozen to the bed, as shown in other tidewater glacier surges (Nuth et al., 2019).

The active surge of Negribreen experiences the greatest flow (change) in the lower part of the glacier, as is shown in Figure 3. Resulting in a terminus advance of a couple of kilometers. Surrounding glaciers have considerably lower velocities and do not seem to be influenced by the surge. The only other fast



Figure 4. Orthorectification bias ( $\delta h$, in equation2) over Negribreen glacier system for April 2018.

flow occurrs on Petermannbreen, a smaller tidewater glacier to the south which is not anymore connected to this glacier system.

The orthorectification bias $(\delta h)$, i.e. elevation differences in respect to the reference elevation model, for the spring of 2018 (Figure 4) provides a strong alternating signal of draw down and elevation increase is present on Negribreen. For the interior of Negribreen a slight but clear negative elevation bias is present. At the glacier snout there is a clear elevation increase, in the location which used to be stagnant ice. The elevation increase is confined to a straight funnel, but a strong positive signal is also present at the calving front of Rembebreen. This sharp contrast does co-align with the coastline before the surge initiated. Other elevation increases can be seen at the snout



Figure 5. Elevation difference between Apr. 2014 Aug. 2017, from ArcticDEM strip data. 
of Petermannbreen and a partial uplift seems to occur on the lower parts of Ordonnansbreen.

These velocity and elevation patterns reflect the mechanisms described by (Nuth et al., 2019), in which a frozen glacier tongue hinders ice transport. After the glacier tongue destabilized in the spring of 2016, elevations have lowered in the accumulation area of Negribreen. However, the surge presently does not seem to influence other glaciers within the same system, except potentially the build-up of stagnant ice on Ordonnabreen. At the confluence of Negribreen with its neighboring Akademikarbreen and Rembebreen, surface slopes will increase due to elevation loss in the upper part of Negribreen. The resulting increase in driving stress might also be an agent to trigger surge instabilities on these glaciers, if this will be the case needs to be seen.

\section{CONCLUSIONS}

In this study we explore the possibility of simultaneous estimation of surface displacement and elevation change. This is accomplished with Sentinel-2 data, a high-repeat medium-resolution pushbroom satellite. The methodology is tested over a glacier in Svalbard, which recently entered a highly dynamic flow regime. Initial results hint towards a slow build-up of mass behind the glacier tongue followed by a sudden release. This caused backward propagation of velocity increases up-glacier, but its influence kept constrained to a narrow outlet. However, at the time of writing processing of the time-series is still happening, and interpretation and checking of our results is still necessary and envisioned. Though our initial results seem trustworthy as independent spaceborne elevation models (Porter et al., 2018) show similar spatial patterns and magnitudes, as can be seen in Figure 5. However, the sign of elevation difference is different for Petermannbreen, this might be due to a difference in timespan between the elevation data which are here compared and associated glacier dynamics prior to 2014. Unfortunately, the elevation data of WorldView is currently not covering the full glacier basin, neither is data available for 2018 , yet.

The photogrammetric set-up of this system was not initially envisioned for Sentinel-2 (Drusch et al., 2012). However, in this study its potential and usefulness is demonstrated for the application of short-term glacier dynamics. It seems this set-up occupies a niche, which is not covered by any other spaceborne system. In this respect it is an improvement over other operational topographic systems, especially in respect to temporal coverage. Even though its precision might not be at the same level as dedicated photogrammetric missions, its continuous sensing makes temporal information extraction possible. Therefor we see this technique as complementary, and similar to timely but medium resolution topographic extraction methods as with the Planet constellation (Ghuffar, 2018).

The advantage of simultaneous extraction of elevation and kinematics over the same period, from the same sensor, increases the functionality of the data for modeling efforts. Typically, temporal alignment of velocity and topographic data is troublesome and additional interpolation is needed, however this is not the case for this integrated set-up. The set-up of this methodology is most suited for High Arctic glaciers, as at these high latitudes overlap is prominent (see Figure 1). Though this technique is still applicable in the lower Arctic, such as mainland Norway and Alaska, as the maximum intersection angle stays constant. This wide angle field of view for Sentinel-2 in combination with its medium resolution pixel resolution is essential for establishing a favorable base-to-height relation. While other popular sun-synchronous systems have less favorable configurations for the demonstrated photogrammetric extraction, as is listed in Table 2.

Another essential element for succes of this implementation is the improved absolute geo-referencing of Sentinel-2, especially in respect to older satellite systems. In our implementation we assume the lateral displacements can be solely attributed to ortho-rectification caused by an outdated elevation model and glacier movement. While for older satellites this co-registration has to be done on neighboring stable terrain (Rosenau et al., 2015, Fahnestock et al., 2016), or through stacking (Altena, Kääb, 2017), which would introduce an additional constrain within our system of equations. Hence given the advanced instrumentation of Sentinel-2, but also its high repeat, wide-angle and medium pixel resolution, this configuration occupies a sweet spot for monitoring of short term glacier dynamics in the Arctic.

\begin{tabular}{|llll|cc|} 
Satellite system & \multicolumn{2}{c}{$\begin{array}{c}\text { Geometric } \\
\text { characteristics }\end{array}$} & \multicolumn{2}{c|}{$\begin{array}{c}\text { Retrieval } \\
\text { precision }\end{array}$} \\
& $\begin{array}{c}\text { Height } \\
{[\mathrm{km}]}\end{array}$ & $\begin{array}{c}\text { Width } \\
{[\mathrm{km}]}\end{array}$ & $\begin{array}{c}\text { Pixel } \\
{[\mathrm{m}]}\end{array}$ & $\begin{array}{c}0.5 \mathrm{px} \\
{[\mathrm{m}]}\end{array}$ & $\begin{array}{c}0.1 \mathrm{px} \\
{[\mathrm{m}]}\end{array}$ \\
\hline
\end{tabular}

Table 2. Expected highest precision achievable for height retrieval through different sun-synchronous satellites.

\section{ACKNOWLEDGEMENTS}

This research is conducted through support from the European Union FP7 ERC project ICEMASS (320816) and the ESA projects Glaciers_cci (4000109873 14 I-NB) and ICEFLOW (4000125560 18 I-NS). Data from Sentinel-2 is made freely available through the Copernicus program and has been essential for this work.

\section{REFERENCES}

Altena, B., Kääb, A., 2017. Elevation change and improved velocity retrieval using orthorectified optical satellite data from different orbits. Remote Sensing, 9, 300.

Altena, B., Scambos, T., Fahnestock, M., Kääb, A., 2019. Extracting recent short-term glacier velocity evolution over Southern Alaska and the Yukon from a large collection of Landsat data. The Cryosphere, 13, 795-814.

Bontemps, N., Lacroix, P., Doin, M-P., 2018. Inversion of deformation fields time-series from optical images, and 
application to the long term kinematics of slow-moving landslides in Peru. Remote Sensing of Environment, 210, $144-158$.

Cogley, J.G., Hock, R., Rasmussen, L.A., Arendt, A.A., Bauder, A., Braithwaite, R.J., Jansson, P., Kaser, G., Möller, M., Nicholson, L., Zemp, M., 2011. Glossary of glacier mass balance and related terms. Technical Report 86, International Association of Cryospheric Sciences, UNESCO-IHP, Paris, France.

Dowdeswell, J.A., Drewry, D.J., Liestøl, O., Orheim, O., 1984. Radio echo-sounding of Spitsbergen glaciers: problems in the interpretation of layer and bottom returns. Journal of Glaciology, 30, 16-21.

Drusch, M., Del Bello, U., Carlier, S., Colin, O., Fernandez, V., Gascon, F., Hoersch, B., Isola, C., Laberinti, P., Martimort, P., Meygret, A., Spoto, F., Sy, O., Marchese, F., Bargellini, P., 2012. Sentinel-2: ESA's optical high-resolution mission for GMES operational services. Remote Sensing of Environment, 120, 25-36.

Eisen, O., Harrison, W.D., Raymond, C.F., Echelmeyer, K.A., Bender, G.A., Gorda, J.L.D., 2005. Variegated Glacier, Alaska, USA: a century of surges. Journal of Glaciology, 51, 399-406.

Fahnestock, M., Scambos, T., Moon, T., Gardner, A., Haran, T., Klinger, M., 2016. Rapid large-area mapping of ice flow using Landsat 8. Remote Sensing of Environment, 185, 84-94.

Fischler, M.A., Bolles, R.C., 1981. Random sample consensus: a paradigm for model fitting with applications to image analysis and automated cartography. Communications of the ACM, 24, 381-395.

Gaudel, A., Languille, F., Delvit, J.M., Michel, J., Cournet, M., Poulain, V., Youssefi, D., 2017. Sentinel-2: Global reference image validation and application to multitemporal performances and high latitude digital surface model. International Archives of the Photogrammetry, Remote Sensing \& Spatial Information Sciences, XLII-1/W1, 447-454.

Ghuffar, S., 2018. DEM generation from multi satellite PlanetScope imagery. Remote Sensing, 10, 1462.

Hadhri, H., Vernier, F., Atto, A., Trouvé, E., 2019. Time-lapse optical flow regularization for geophysical complex phenomena monitoring. ISPRS Journal of Photogrammetry and Remote Sensing, 150, 135-156.

Hamilton, G.S., Dowdeswell, J.A., 1996. Controls on glacier surging in Svalbard. Journal of Glaciology, 42, 157-168.

Kääb, A., Winsvold, S.H., Altena, B., Nuth, C., Nagler, T., Wuite, J., 2016. Glacier remote sensing using Sentinel-2. part I: Radiometric and geometric performance, and application to ice velocity. Remote Sensing, 8, 598.

Kaufmann, V., Ladstädter, R., 2004. Documentation of the movement of the Hinteres Langtalkar rock glacier. International Archives of Photogrammetry and Remote Sensing, 35, 893-898.

Lacroix, P., Bièvre, G., Pathier, E., Kniess, U., Jongmans, D., 2018. Use of Sentinel-2 images for the detection of precursory motions before landslide failures. Remote Sensing of Environment, 215, 507-516.
Lefauconnier, B., Hagen, J.O., 1991. Surging and calving glaciers in eastern Svalbard. Meddelelser, 116, Norwegian Polar Institute.

Leprince, S., Barbot, S., Ayoub, F., Avouac, J-P., 2007. Automatic and precise orthorectification, coregistration, and subpixel correlation of satellite images, application to ground deformation measurements. IEEE Transactions on Geoscience and Remote Sensing, 45, 1529-1558.

Li, R., Ye, W., Qiao, G., Tong, X., Liu, S., Kong, F., Ma, X., 2017. A new analytical method for estimating Antarctic ice flow in the 1960s from historical optical satellite imagery. IEEE Transactions on Geoscience and Remote Sensing, 55, 2771-2785.

Nuth, C., Gilbert, A., Köhler, A., McNabb, R., Schellenberger, T., Sevestre, H., Weidle, C., Girod, L., Luckman, A., Kääb, A., 2019. Dynamic vulnerability revealed in the collapse of an Arctic tidewater glacier. Scientific Reports, to appear.

Ottesen, D., Dowdeswell, J.A., Bellec, V.K., Bjarnadóttir, L.R., 2017. The geomorphic imprint of glacier surges into open-marine waters: examples from eastern Svalbard. Marine Geology, 392, 1-29.

Porter, C., Morin, P., Howat, I., Noh, M-J., Bates, B., Peterman, K., Keesey, S., Schlenk, M., Gardiner, J., Tomko, K., Willis, M., Kelleher, C., Cloutier, M., Husby, E., Foga, S., Nakamura, H., Platson, M., Wethington, M., Williamson, C., Bauer, G., Enos, J., Arnold, G., Kramer, W., Becker, P., Doshi, A., DSouza, C., Cummens, P., Laurier, F., Bojesen, M., 2018. ArcticDEM. Harvard Dataverse, V1. doi.org/10.7910/DVN/OHHUKH.

Raymond, C.F., 1987. How do glaciers surge? A review. Journal of Geophysical Research: Solid Earth, 92, 9121-9134.

Ressl, C., Pfeifer, N., 2018. Evaluation of the elevation model influence on the orthorectification of Sentinel-2 satellite images over Austria. European Journal of Remote Sensing, 51, 693-709.

Rosenau, R., Scheinert, M., Dietrich, R., 2015. A processing system to monitor Greenland outlet glacier velocity variations at decadal and seasonal time scales utilizing the Landsat imagery. Remote sensing of environment, 169, 1-19.

Strozzi, T., Paul, F., Wiesmann, A., Schellenberger, T., Kääb, A., 2017. Circum-Arctic changes in the flow of glaciers and ice caps from satellite SAR data between the 1990s and 2017. Remote Sensing, 9, 947.

Sund, M, Eiken, T, Hagen, JO, Kääb, A, 2009. Svalbard surge dynamics derived from geometric changes. Annals of Glaciology, 50, 50-60.

Westerweel, J., Scarano, F., 2005. Universal outlier detection for PIV data. Experiments in fluids, 39, 1096-1100. 